\title{
On the non-Gaussianity of chain motion in unentangled polymer melts
}

\author{
Grant D. Smith \\ Department of Materials Science and Engineering, 122 S. Central Campus Drive Room 304, \\ University of Utah, Salt Lake City, Utah 84112 \\ Wolfgang Paul \\ Institut für Physik, Johannes-Gutenberg Universität, 55099 Mainz, Germany
}

M. Monkenbusch and D. Richter

Institut für Festkörperforschung, Forschungszentrum Jülich, 52425 Jülich, Germany

(Received 21 August 2000; accepted 18 December 2000)

\begin{abstract}
We have investigated chain dynamics of an unentangled polybutadiene melt via molecular dynamics simulations and neutron spin echo experiments. Good short-time statistics allows for the first experimental confirmation of subdiffusive motion of polymer chains for times less than the Rouse time $\left(\tau_{R}\right)$ confirming behavior in this regime observed in simulations. Analysis of simulation trajectories obtained over several Rouse times reveals non-Gaussian segmental displacements for all time and length scales. These results, particularly non-Gaussian displacements on large time- and length scales, demonstrate the importance of intermolecular correlations on chain dynamics. Rouse-type analytical models fail to account for this non-Gaussianity leading to large deviations between the experimental dynamic structure factor and model predictions. (C) 2001 American Institute of Physics. [DOI: 10.1063/1.1348032]
\end{abstract}

\section{INTRODUCTION}

Recently, ${ }^{1}$ we demonstrated excellent agreement between molecular dynamics (MD) simulations and neutron spin echo (NSE) studies for chain dynamics in an unentangled melt of $\mathrm{C}_{100} \mathrm{H}_{202}$ polyethylene (PE), allowing for critical testing of Rouse predictions for real polymer chains. The Rouse model is commonly invoked in the interpretation of experimental data for polymer melts, and forms the basis of many dynamic theories including reptation theories for entangled melts. The Rouse model failed to provide an accurate description of the dynamic structure factor $S(q, t)$ of the PE melt. Based upon subdiffusive behavior of the polymer chains observed in the simulations, we attributed this failure to intermolecular correlations. A recent theoretical study has also found that interactions of a chain in the melt with $\sqrt{N}$ other chains leads to subdiffusive behavior for times less than the Rouse time $\tau_{R} .{ }^{2}$ The importance of intermolecular correlations in the failure of the Rouse model has been refuted in a study that claimed that a semiflexible chain model (SFCM) that accounts for local stiffness effects yields much better agreement with simulation for $S^{\prime}(q, t)$ of the PE melt than the Rouse model. ${ }^{3}$ In another attempt to improve on the Rouse model, Allegra and Ganazzoli ${ }^{4,5}$ have developed a model that includes internal friction effects that slow down short wavelength motions. The "internal viscosity" model (IVM) has been shown to provide a good description of the dynamic structure factor for polyisobutylene (PIB) when internal friction effects are taken into account. ${ }^{6}$ Clearly, the ability of simple analytical treatments to reproduce the dynamics of unentangled polymer melts remains at issue. We believe that we now have experimental and simulation data of sufficient accuracy to resolve this important question.

\section{EXPERIMENTS AND SIMULATIONS}

NSE measurements and MD simulations were performed on an unentangled 1,4-polybutadiene (PBD) melt with a random microstructure of 30 repeat units consisting of $40 \%$ cis, $50 \%$ trans, and 10\% 1,2-vinyl units. Both experimental and simulation data represent a significant improvement over those presented in our previous work ${ }^{7}$ as the result of additional measurements and simulations. Special effort has been expended to improve the statistical reliability of the NSE data, particularly at short times. The MD trajectory has been greatly extended to almost three Rouse times in order to improve long-time statistics. NSE measurements were performed on the NSE spectrometer at the FRJ-2 reactor in Jülich at $353 \mathrm{~K}$, studying spectra at seven different momentum transfers $0.05 \AA^{-1} \leqslant q \leqslant 0.30 \AA^{-1}$ covering a dynamic range from 100 ps to $22 \mathrm{~ns}$. Data analysis was performed as described previously. ${ }^{7}$ MD simulations were performed using a quantum chemistry-based united atom potential ${ }^{7,8}$ using methods described previously. ${ }^{7,8}$ The isotropic melt single chain intermediate coherent dynamic structure factor measured in NSE experiments corresponds to ${ }^{9}$

$$
\begin{aligned}
S^{\prime}(q, t)= & S(q, t) / S(q) \\
= & \sum_{(m, n)}\left\langle\sin \left[q R_{\mathrm{mn}}(t)\right] / q R_{\mathrm{mn}}(t)\right\rangle / \\
& \sum_{(m, n)}\left\langle\sin \left[q R_{\mathrm{mn}}(0)\right] / q R_{\mathrm{mn}}(0)\right\rangle,
\end{aligned}
$$




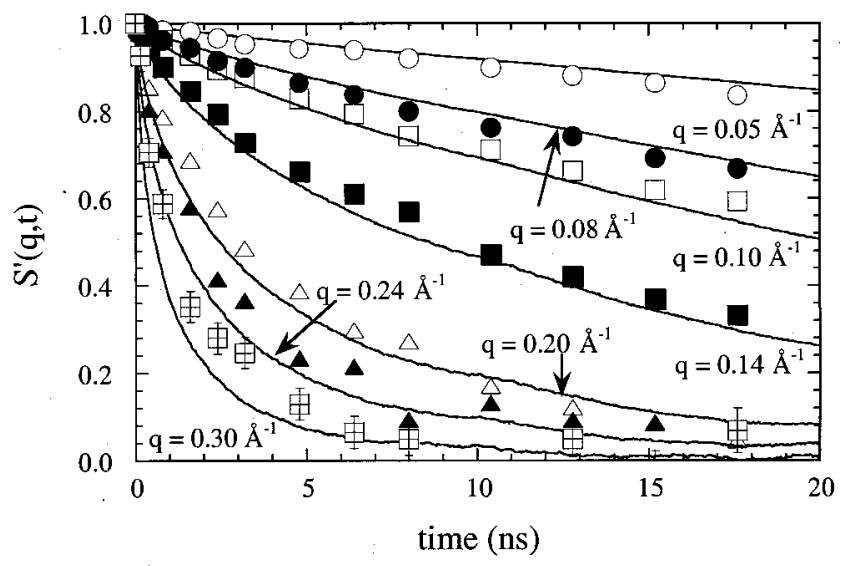

FIG. 1. Dynamic structure factor for PBD chains obtained from simulation (lines) and neutron spin echo measurements (symbols). Error bars (random errors) are shown for $q=0.30 \AA^{-1}$. For all other data estimated random errors are smaller than the symbols.

where $R_{\mathrm{mn}}(t)$ is the magnitude of the displacement vector $\mathbf{R}_{m}(t)-\mathbf{R}_{n}(0)$ between scattering centers $m$ and $n$ at time $t$ and zero, repectively, with $m$ and $n$ belonging to the same chain. When $q \ll 2 \pi / R_{g}$, one can observe only the overall center of mass motion $\left\langle R_{\mathrm{cm}}(t)^{2}\right\rangle$ of the chain molecules, and assuming $\left\langle R_{\mathrm{cm}}(t)^{2}\right\rangle$ is diffusive,

$$
S^{\prime}(q, t)=\exp \left(-q^{2}\left\langle R_{\mathrm{cm}}(t)^{2}\right\rangle / 6\right)=\exp \left(-q^{2} D_{\mathrm{cm}} t\right),
$$

where $D_{\mathrm{cm}}$ is the center of mass self-diffusion coefficient for the chains.

Good agreement between $S^{\prime}(q, t)$ from NSE measurements and simulation can be seen in Fig. 1. The experimental times have been scaled by 0.8 to compensate for the very small difference (20\%) in the apparent $D_{\mathrm{cm}}$ [Eq. (2)] between simulation and experiment. It is possible to calculate an apparent mean-square center of mass displacement $\left\langle R_{\mathrm{cm}}(t)^{2}\right\rangle_{\text {app }}$ of the chains from Eq. (2). $\left\langle R_{\mathrm{cm}}(t)^{2}\right\rangle_{\text {app }}$ obtained from experimental $S^{\prime}(q, t)$ data (unscaled) are compared in Fig. 2 with $\left\langle R_{\mathrm{cm}}(t)^{2}\right\rangle$ obtained directly from simulation. For $t>\tau_{R}=15 \mathrm{~ns}$, the center-of-mass motion is well

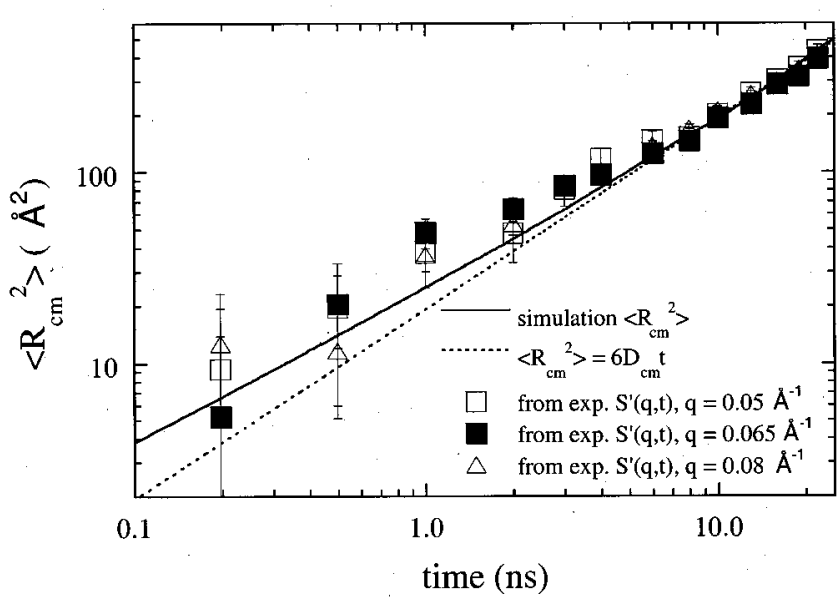

FIG. 2. Mean-square center-of-mass displacement of PBD chains. Errors bars (random errors) are show for the experimental data.

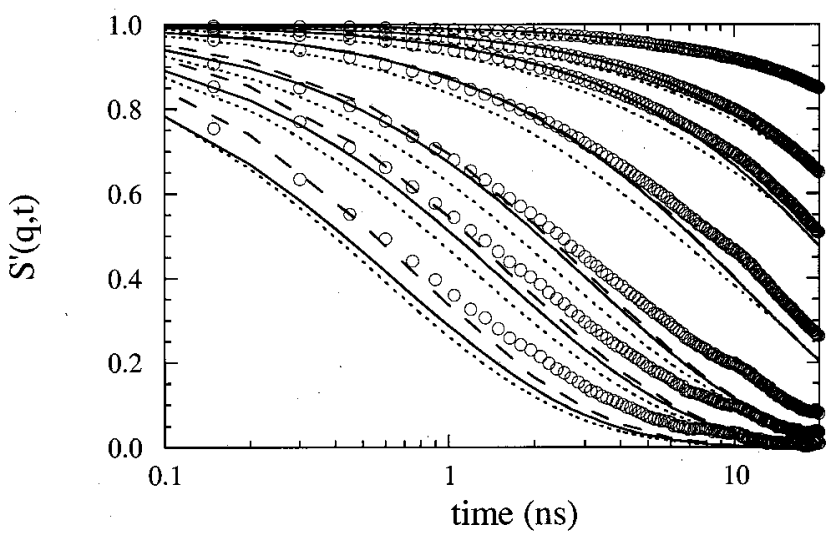

FIG. 3. Dynamic structure factor for PBD chains obtained from simulation (symbols), the Rouse model (solid lines), the modified Rouse model (dotted lines), and the semiflexible chain model (dashed lines). The $q$ values are the same as shown in Fig. 1.

described by $\left\langle R_{\mathrm{cm}}(t)^{2}\right\rangle=6 D_{\mathrm{cm}} t$. For $t<\tau_{R}$, the motion is subdiffusive. Here, $\left\langle R_{\mathrm{cm}}(t)^{2}\right\rangle \sim t^{a}$ where $a \approx 0.8$, as was observed in our simulations of $\mathrm{PE}^{1}$ and earlier simulations of coarse-grained lattice ${ }^{10}$ and bead-spring models. ${ }^{11}$ The experimental data for PBD are of sufficient quality to confirm for the first time subdiffusive behavior of polymer chains for $t<\tau_{R}$, particularly when considered in conjunction with the observed $\left\langle R_{\mathrm{cm}}(t)^{2}\right\rangle$ from simulation.

\section{SIMULATIONS AND THEORY}

The good agreement between simulation and experiment for $S^{\prime}(q, t)$ allows us to investigate the validity of the Rouse model, ${ }^{12}$ the SFCM, ${ }^{3,13}$ and the IVM $^{4,5}$ in describing real polymer dynamics through detailed comparison of the predictions of these models with simulations. In these models polymer segments are subjected to internal entropic restoring forces and a frictional drag force and move in response to Brownian forces. The models neglect excluded volume and hydrodynamic effects and predict diffusive center-of-mass motion for all times. The restoring forces are determined by chain dimensions $[S(q)]$. In the Rouse model, the chain is assumed Gaussian on all length scales, while in the SFCM (adjustable) local stiffness effects yield non-Gaussian behavior for large $q$. In the IVM, an approximate analytical $S(q)$ is employed. In both the Rouse model and SFCM, the frictional drag force is assumed to be independent of wavelength, while in the IVM local friction effects slow short wavelength motions.

The solution of the Rouse equation of motion is determined by transformation to its eigenmodes, whose selfcorrelation functions are given as ${ }^{9}$

$$
\left\langle\mathbf{X}_{p}(t) \cdot \mathbf{X}_{p}(0)\right\rangle=\frac{\left\langle R^{2}\right\rangle}{2 \pi^{2} p^{2}} \exp \left[-p^{2} t / \tau_{R}\right] .
$$

Simulations yield $\tau_{R}=\left\langle R^{2}\right\rangle /\left(3 \pi^{2} D_{\mathrm{cm}}\right)=15 \mathrm{~ns}$ for PBD. Figure 3 gives a comparison of $S^{\prime}(q, t)$ from simulation and the Rouse model. As for PE, ${ }^{1}$ the Rouse model does a poor job in reproducing simulation. A detailed analysis of the simulation trajectories allows us to investigate the fundamental pre- 


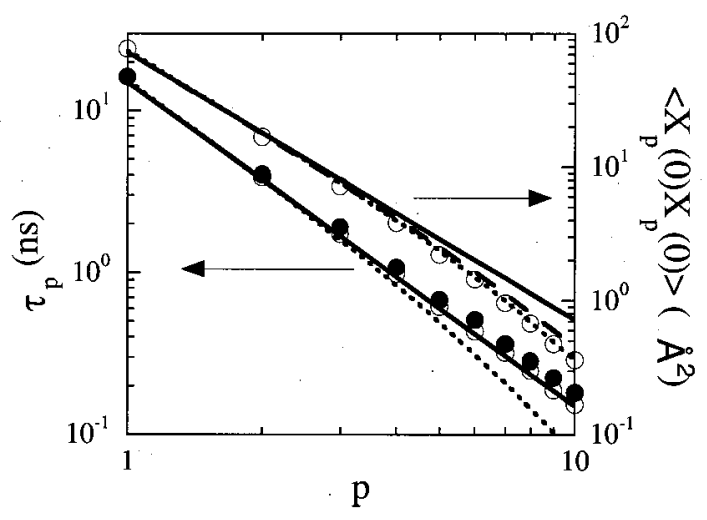

FIG. 4. Normal mode amplitude and relaxation time for PBD chains from simulation (symbols, with filled circles being correlation times), the Rouse model (solid lines), the semiflexible chain model (dotted lines), and the internal viscosity model (dashed line).

dictions of the Rouse model. The amplitudes and relaxation times for the important eigenmodes are compared in Fig. 4. The amplitudes from simulation for modes $p>3$ begin to show deviation from Rouse predictions as $R_{m n}(0)$ are not Gaussian distributed for small $|n-m|$ due to local chain stiffness effects. The mode self-correlation functions from simulation do not show single exponential decay, but are well represented by a stretched exponential

$$
\left\langle\mathbf{X}_{p}(t) \cdot \mathbf{X}_{p}(0)\right\rangle=\left\langle\mathbf{X}_{p}(0) \cdot \mathbf{X}_{p}(0)\right\rangle \exp \left[-\left(t / \tau_{p}\right)^{\beta}\right],
$$

where $\beta$ ranges from 1.0 for $p=1$ to 0.75 for $p=10$. Correlation times, given as the time integral of $\exp \left[-\left(t / \tau_{p}\right)^{\beta}\right]$, are also shown in Fig. 4. The shorter wavelength (large $p$ ) modes show some slowing relative to Rouse predictions. Relaxation times clearly do not scale with mode amplitudes as is predicted by the Rouse model. We modified the relationship for $S^{\prime}(q, t)$ for the Rouse model ${ }^{9}$ to take into account $\left\langle R_{\mathrm{cm}}(t)^{2}\right\rangle$, mode amplitudes $\left\langle\mathbf{X}_{p}(0) \cdot \mathbf{X}_{p}(0)\right\rangle$ as well as relaxation times $\tau_{p}$ and stretching exponents $\beta$ from simulation. As shown in Fig. 3, the resulting $S^{\prime}(q, t)$ decays faster than predicted by the Rouse model due to the fact that $\left\langle R_{\mathrm{cm}}(t)^{2}\right\rangle$ is greater in the subdiffusive regime than predicted by the Rouse model (Fig. 2). The modified Rouse and Rouse predictions merge at $\tau_{R}$, where the center-of-mass displacement becomes diffusive. Clearly, incorporating differences in mode amplitudes and relaxation into the Rouse predicted scattering function is not sufficient to account for the large discrepancies observed in $S^{\prime}(q, t)$ between the Rouse model and simulations.

In the SFCM the contour length $L_{c}$ and the persistence length $L_{p}$ are adjustable parameters. Using the relationships for a Kratky-Porod chain, structural data for PBD from simulation establishes values of $L_{p}=5.0 \AA$ and $L_{c}=147 \AA$, yielding $\left\langle R^{2}\right\rangle /\left\langle R_{g}^{2}\right\rangle=1420 \AA^{2} / 221 \AA^{2}=6.4$, while from simulations $\left\langle R^{2}\right\rangle /\left\langle R_{g}^{2}\right\rangle=1414 \AA^{2} / 224 \AA^{2}=6.3$. Use of significantly stiffer semiflexible chains as was done for PE in order to achieve the claimed good agreement of the model with experiment and simulation does not reproduce the static structure of the chain on longer length scales. Predictions of $S^{\prime}(q, t)$ for the SFCM with $D_{\mathrm{cm}}=3.2 \times 10^{-7} \mathrm{~cm}^{2} / \mathrm{s}$ taken from simulation are shown in Fig. 3. The corresponding mode amplitudes and relaxation times are given in Fig. 4. Mode amplitudes are in good agreement with those from simulation, and are reduced from the Rouse amplitudes, indicating the influence of local chain stiffness. The corresponding relaxation times, as with the Rouse model, are proportional to the mode amplitude, and hence are shorter than those predicted by the Rouse model, and are in poor agreement with simulation for larger $p$. This variance becomes greater with increasing chain stiffness. $S^{\prime}(q, t)$ obtained from the SFCM shows little improvement over the Rouse model. At long times, SFCM and Rouse predictions are indistinguishable. At short times, the decay of $S^{\prime}(q, t)$ from the SFCM is slower than that seen in the simulations or the Rouse model. We again modified the relationship for $S^{\prime}(q, t)$ for the Rouse model, this time using mode amplitudes from the SFCM. Comparison of modified Rouse and SCFM predictions reveals that the effect of amplitude reduction cannot account for the reduced decay in $S^{\prime}(q, t)$ at short time seen with the SFCM. This reduction, which becomes greater with increasing chain stiffness, must therefore be due to the nonGaussian distribution of distances within the semiflexible chain.

The mode amplitudes from the IVM are shown in Fig. 4 and are in good agreement with simulation and predictions of the SFCM. Unlike PIB,${ }^{6}$ where a fit of the IVM to experimental data indicated a dramatic increase in relaxation time for short wavelength modes, a fit to experiment for PBD yields relaxation times and hence $S^{\prime}(q, t)$ similar to those of the SFCM. Hence, in agreement with simulation, internal friction effects do not appear to be important in PBD.

Summarizing, we see that: (1) atomistic MD simulations of unentangled polymer melts using quantum chemistrybased potentials yield $S^{\prime}(q, t)$ in good agreement with NSE measurements; (2) the Rouse model does a poor job in reproducing $S^{\prime}(q, t)$ from simulation and experiment; (3) the failure of the Rouse model does not lie primarily in the predicted mode amplitudes or relaxation times; (4) efforts to improve upon the Rouse model by including local stiffness effects using the correct static structure of the chain do not lead to a significantly improved description of $S^{\prime}(q, t) ;(5)$ a dramatic increase in relaxation times for short wavelength modes compared to Rouse predictions is inconsistent with simulations and IVM predictions. The question therefore remains as to why the models fail to accurately reproduce $S^{\prime}(q, t)$.

\section{NON-GAUSSIANITY}

Calculation of $S^{\prime}(q, t)$ for these models is based upon the assumptions that all segmental displacements $\mathbf{R}_{m}(t)$ $-\mathbf{R}_{n}(0)$ are Gaussian distributed and that the eigenmodes remain orthogonal, i.e., $\left\langle\mathbf{X}_{r}(t) \cdot \mathbf{X}_{s}(0)\right\rangle=0$ for $r \neq s$. Examination of cross correlation for the $p \leqslant 4$ from simulation confirms that the modes remain orthogonal. Let us then consider the assumption of Gaussian distributed displacements. $S^{\prime}(q, t)$ for isotropic systems is given by Eq. (1). Only when $\mathbf{R}_{m}(t)-\mathbf{R}_{n}(0)$ are Gaussian distributed should the Gaussian approximation be invoked, allowing Eq. (1) to be recast as 


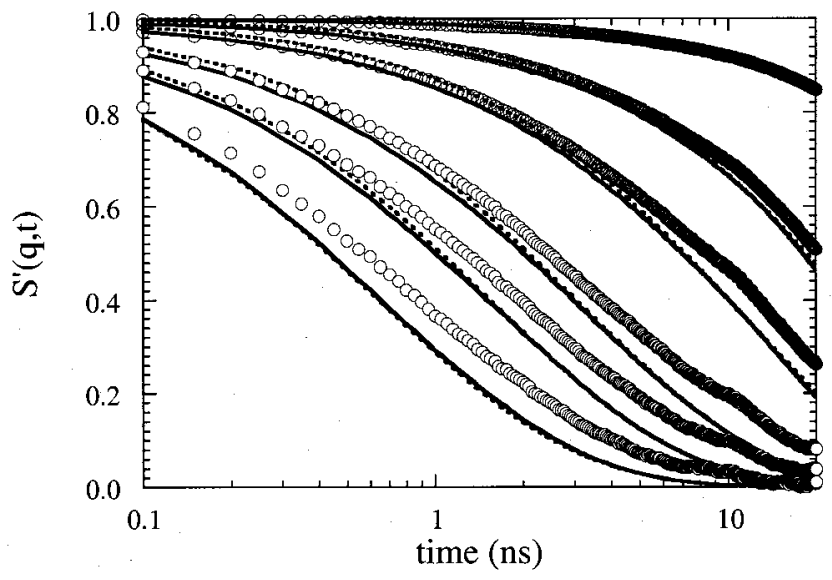

FIG. 5. Comparison of the dynamic structure factor for PBD chains obtained from simulation using Eq. (1) (symbols) and Eq. (5) (solid lines), $q$ $=0.08 \AA^{-1}$ are not shown for clarity. Also shown are the Rouse predictions (dotted lined). The $q$ values are the same as shown in Fig. 1 expect that the $q=0.08 \AA^{-1}$ data have been omitted for clarity.

$$
\begin{aligned}
S^{\prime}(q, t)= & \sum_{(m, n)} \exp \left[-q^{2}\left\langle R_{\mathrm{mn}}(t)^{2}\right\rangle / 6\right] / \\
& \sum_{(m, n)} \exp \left[-q^{2}\left\langle R_{\mathrm{mn}}(0)^{2}\right\rangle / 6\right]=S(q, t) / S(q) .
\end{aligned}
$$

Since solution of the dynamic equation for each analytical model yields $\left\langle R_{\mathrm{mn}}(t)^{2}\right\rangle$, Eq. (5) is conveniently employed in determining $S^{\prime}(q, t)$. However, assumption of Gaussian distributed displacements is valid only for the Rouse model. Indeed, a primary goal of the SFCM and IVM is to account for the effects of chain stiffness on dynamics. Use of Eq. (5) is simply invalid for all models considered here except the Rouse model.

We can get a picture of the influence of non-Gaussian distributed displacements on $S^{\prime}(q, t)$ by using both Eq. (1) and Eq. (5) to predict $S^{\prime}(q, t)$ for simulation chains, as shown in Fig. 5. $S^{\prime}(q, t)$ yielded by Eq. (5) for the simulation chains are very similar to the Rouse predictions as shown in Fig. 5. This indicates that the mean-square displacements on all time and length scales investigated are reasonably well described by the Rouse model. In contrast, the variance in $S^{\prime}(q, t)$ between the SFCM and both simulation using Eq. (5) and the Rouse model at short times and larger $q$ values indicates that the SFCM chain is too stiff on the corresponding length scales. However, we see in Fig. 3 that $S^{\prime}(q, t)$ for the SFCM converges to the Rouse predictions after sufficient time. For the SFCM, all particle selfdisplacements $\mathbf{R}_{m}(t)-\mathbf{R}_{m}(0)$ are Gaussian distributed, so eventually $\mathbf{R}_{m}(t)-\mathbf{R}_{n}(0)$ and $S^{\prime}(q, t)$ for the SFCM converge with those for the Gaussian chain, i.e., the Rouse model. In contrast, $S^{\prime}(q, t)$ from simulations using Eq. (1) do not converge with those from Eq. (5), even for $t \approx \tau_{R}$, clearly demonstrating that the displacements are not Gaussian distributed even on this time scale.
From the MD trajectories we can examine the displacement distributions as a function of $|n-m|$ and time. We find that even after one Rouse time $\mathbf{R}_{m}(t)-\mathbf{R}_{n}(0)$ distributions show deviation from Gaussian behavior for all $|n-m|$. The influence of these non-Gaussian displacements on $S^{\prime}(q, t)$ depends upon $q, t$ and $|n-m|$. However, non-Gaussian effects will in all cases slow the decay of $S^{\prime}(q, t)$ relative to that obtained for a Gaussian distribution with the same mean-square displacements (e.g., see Fig. 5), consistent with the observed relationship between simulation and Rouse predictions. For example, the contribution of center-of-mass displacement to $S^{\prime}(q, t)$ with the first non-Gaussian correction is given by ${ }^{14}$

$$
\begin{aligned}
S_{\mathrm{cm}}^{\prime}(q, t)= & \exp \left[-q^{2}\left\langle R_{\mathrm{cm}}(t)^{2}\right\rangle / 6\right] \\
& \times\left[1+1 / 2\left[q^{2}\left\langle R_{\mathrm{cm}}(t)^{2}\right\rangle / 6\right]^{2} \alpha_{2}(t)\right],
\end{aligned}
$$

where

$$
\alpha_{2}(t)=\frac{3\left\langle R_{\mathrm{mn}}(t)^{4}\right\rangle}{5\left\langle R_{\mathrm{mn}}(t)^{2}\right\rangle^{2}}-1 .
$$

For $t=15 \mathrm{~ns}$ and $q=0.30 \AA^{-1}$, simulations yield $\alpha_{2}(t)$ $=0.15$ and $1 / 2\left[q^{2}\left\langle R_{\mathrm{cm}}(t)^{2}\right\rangle / 6\right]^{2} \alpha_{2}(t)=1.37$, clearly demonstrating the importance of non-Gaussian displacements. NonGaussian displacements for the center of mass and large $\mid n$ $-m \mid$ after times comparable to $\tau_{R}$ can only result from nondiffusive behavior of the center-of-mass displacement and long wavelength modes such as the rotational diffusion of the molecule. Nondiffusive behavior for these modes results from intermolecular correlations, which are not included in any of the models considered except simulations.

Hence, it is primarily the non-Gaussian selfdisplacements $\mathbf{R}_{m}(t)-\mathbf{R}_{m}(0)$ resulting from intermolecular correlations that account for the deviation of Rouse predictions from simulation. Inclusion of chain stiffness does not significantly improve the description. Reasonable agreement with real chain dynamics claimed for the analytical models discussed here results from incorrect calculation of $S^{\prime}(q, t)$ and the use of artificially stiff chains.

${ }^{1}$ W. Paul, G. D. Smith, D. Y. Yoon, B. Farago, S. Rathgeber, A. Zirkel, L. Willner, and D. Richter, Phys. Rev. Lett. 80, 2346 (1998).

${ }^{2}$ M. Guenza, J. Chem. Phys. 110, 7574 (1999).

${ }^{3}$ L. Harnau, R. G. Winkler, and P. Reineker, Phys. Rev. Lett. 82, 2408 (1999).

${ }^{4}$ G. Allegra and F. J. Ganazzoli, J. Chem. Phys. 74, 1310 (1981).

${ }^{5}$ G. Allegra and F. J. Ganazzoli, Macromolecules 14, 1110 (1981).

${ }^{6}$ D. Richter, M. Monkenbusch, J. Allgeier, A. Arbe, J. Colmenero, B. Farago, Y. C. Bae, and R. J. Faust, J. Chem. Phys. 111, 6107 (1999).

${ }^{7}$ G. D. Smith, W. Paul, M. Monkenbusch, L. Willner, D. Richter, X. H. Qiu, and M. D. Ediger, Macromolecules 32, 8857 (1999).

${ }^{8}$ G. D. Smith and W. Paul, J. Phys. Chem. A 102, 1200 (1998).

${ }^{9}$ M. Doi and S. F. Edwards, The Theory of Polymer Dynamics (Cornell University Press, Ithaca, NY, 1979).

${ }^{10}$ W. Paul, K. Binder, D. W. Heermann, and K. Kremer, J. Chem. Phys. 95, 7726 (1991).

${ }^{11}$ A. Kopf, B. Dünweg, and W. Paul, J. Chem. Phys. 107, 6945 (1997).

${ }^{12}$ P. E. Rouse, J. Chem. Phys. 21, 1273 (1955).

${ }^{13}$ L. Harnau, R. G. Winkler, and P. Reineker, J. Chem. Phys. 104, 6355 (1996).

${ }^{14}$ W. van Megen, T. C. Mortensen, S. R. Williams, and J. Müller, Phys. Rev. E 58, 6073 (1998) 